\title{
O papel do enfermeiro no ciclo gravídico-puerperal frente à violência obstétrica: uma revisão integrativa
}

\author{
The role of nurses in the pregnancy-puerperal cycle in the face of obstetric violence: an \\ integrative review
}
El papel de las enfermeras en el ciclo embarazo-puerperal ante la violencia obstétrica: una revisión integradora

Jéssica Pereira Soares de Souza ${ }^{1 *}$, Leticia Stefany Dantas Santos ${ }^{1}$, Mayara Cabral de Freitas ${ }^{1}$, Lidiane Bernardino Andrade de Carvalho Virginio ${ }^{1}$, Flávia Roberta de Souza ${ }^{1}$, Eduardo Silva Gomes de Araujo ${ }^{1}$, Hirla Vanessa Soares de Araújo².

\section{RESUMO}

Objetivo: Buscar na literatura o que as evidências científicas abordam sobre os tipos de violências obstétricas e o papel do enfermeiro. Métodos: Trata-se de uma revisão integrativa da literatura, de caráter exploratório e cunho qualitativo Foram utilizados os descritores: "assistência de enfermagem", "prática de enfermagem" combinados às palavras: "violência obstétrica", selecionando artigos publicados no período de 2015 a 2021 na base de dados Medline, LILACS e Acervo+. A busca resultou em 19 artigos. Após a utilização dos critérios de inclusão e exclusão, o corpus da análise resultou em 11 artigos. Resultados: Após leitura e análise dos artigos relacionados, foi identificado que muitas mulheres não têm ciência sobre a prática de violências a que são submetidas por considerarem atos normais, quando na verdade trata-se de violências obstétricas e que o papel do enfermeiro nos detalhes é essencial para a mudança desses atos. Considerações finais: Visto que é de suma importância mudanças no modelo de assistência obstétrica no Brasil, há uma grande necessidade de expor para as parturientes a importância de se manterem informadas sobre a violência obstétrica, sendo orientadas e tratadas como donas do próprio corpo.

Palavras-chave: Violência obstétrica, Assistência de enfermagem, Prática de enfermagem.

\begin{abstract}
Objective: To search in the literature what scientific evidence addresses about the types of obstetric violence and the role of nurses. Methods: This is an integrative literature review, exploratory and qualitative. The following descriptors were used: "nursing care", "nursing practice" combined with the words: "obstetric violence", selecting articles published in the period from 2015 to 2021 in the Medline, LILACS and Acervo+ database. The search resulted in 19 articles. After using the inclusion and exclusion criteria, the corpus of the analysis resulted in 11 articles. Results: After reading and analyzing the related articles, it was identified that many women are not aware of the practice of violence to which they are subjected because they consider normal acts, when in fact it is obstetric violence and that the role of nurses in details are essential for the change of these acts. Final considerations: Since changes in the obstetric care model in Brazil are of paramount importance, there is a great need to expose to parturients the importance of keeping informed about obstetric violence, being guided and treated like owners of their own body.
\end{abstract}

Key words: Obstetric violence, Nurse, Childbirth.

\section{RESUMEN}

Objetivo: Buscar en la literatura qué evidencia científica aborda sobre los tipos de violencia obstétrica y el papel de las enfermeras. Métodos: Se trata de una revisión bibliográfica integradora, exploratoria y cualitativa. Se utilizaron los siguientes descriptores: "cuidados de enfermería", "práctica de enfermería" combinados con las palabras: "violencia obstétrica", seleccionando artículos publicados en el período de 2015 a 2021 en la

\footnotetext{
${ }^{1}$ Centro Universitário dos Guararapes (UNIFG), Jaboatão dos Guararapes - PE.

*E-mail: jessicasouzaps29@gmail.com

2 Universidade de Pernambuco (UPE), Recife - PE.
} 
Medline, Base de datos LILACS y Acervo+. La búsqueda resultó en 19 artículos. Después de utilizar los criterios de inclusión y exclusión, el corpus del análisis resultó en 11 artículos. Resultados: Tras leer y analizar los artículos relacionados, se identificó que muchas mujeres no son conscientes de la práctica de violencia a la que son sometidas porque consideran actos normales, cuando en realidad se trata de violencia obstétrica y que el papel de la enfermera en los detalles son esenciales para el cambio de estos actos. Consideraciones finales: Dado que los cambios en el modelo de atención obstétrica en Brasil son de suma importancia, existe una gran necesidad de exponer a las parturientas la importancia de mantenerse informadas sobre la violencia obstétrica, ser guiadas y tratadas como dueñas de su propio cuerpo.

Palabras clave: Violencia obstétrica, Cuidados de enfermería, Práctica de enfermería.

\section{INTRODUÇÃO}

Durante o processo de parto, inúmeras mulheres são vítimas de abusos e tratamentos desrespeitosos no âmbito das instituições de saúde. Essa realidade atinge diversos países, o que acaba gerando diversos traumas tanto físicos como mental (ZANARDO GLP, et al., 2017).

Atualmente, o parto vaginal é associado a muita dor e sofrimento por conta do modelo de assistência obstétrica definido como tecnocrático onde prega a hospitalização, visão de um evento patológico com necessidade de condução medica no parto, iniciado com o aumento da tecnologia a partir do século XX (BRANDT GP, et al., 2017).

Para Brandt GP, et al. (2017), Violência Obstétrica (VO) é utilizado para se caracterizar todos os tipos de violências a qual as mulheres venham a sofrer no decorrer da gestação, parto, pós parto ou até mesmo em abortamentos. Vários são os tipos de agressões: verbal, física, moral, psicológica e institucional. São caracterizadas VO falta de acesso a saúde e locomoção de hospital em hospital em busca de atendimento, negligências no processo de assistência, interversões desnecessárias até como a realização da cesariana sem real indicação sendo considerado um perigo para a mãe e seu bebê.

Com o tempo houve mudanças na forma de " dar à luz" como os diversos tipos de partos: cesáreo, fórceps, natural. No entanto, são de grande importância a utilização de técnicas assépticas e é essencial a presença de um profissional capacitado como médico e/ou um enfermeiro obstétrico para auxiliar e acompanhar durante a preparação da mulher. Nesse processo, condutas que na maioria das vezes apenas são usadas para acelerar o parto, como administração de medicamentos e manobras por vezes são realizadas e acaba contribuindo para a desumanização do parto e abre o caminho para a VO (MOURA RCM, et al., 2019).

Para Zanardo GLP, et al. (2017), há práticas realizadas durante o processo que são consideradas desnecessárias por serem desagradáveis e causarem dor, além das mencionadas. Tratam-se de práticas que envolvem a obrigatoriedade da tricotomia de pêlos na região íntima, episiotomias, enemas e impedimento do acompanhante durante $o$ trabalho de parto, práticas essas que não são baseada em evidências cientificas. As intervenções em excesso fizeram com que esquecesse o processo do parto que vai além de doença e morte, é algo particular que precisa considerar os aspectos emocionais, humanos e culturais (MINISTÉRIO DA SAÚDE, 2016).

Um cuidado que deve ser realizado envolve a orientação da gestante sobre todos seus direitos, entre eles o direito a ter acompanhante, seja membro da família ou companheiro, durante o trabalho de parto e parto. Tal conduta é fundamental para que a mulher se sinta segura. O acompanhante deve estar presente nas consultas de pré-natal para e ser orientado pelo profissional de enfermagem sobre a VO, para que o mesmo compreenda e ajude a prevenir e denunciar qualquer tipo de violência que venha a ocorrer (COSTA NY, et al., 2020).

O papel do enfermeiro obstetra reflete grande benefício para esse tipo de assistência. De acordo com estudos com outros profissionais, o olhar humanizado e integral do profissional enfermeiro, buscando as reais necessidades da mulher, é fundamental e vem a minimizar a utilização de técnicas invasivas (LEMOS TAB, et al., 2019). 
Nesse contexto Moura RCM, et al. (2019), em relação aos fatores de risco para a ocorrência da violência obstétrica, é de suma importância que o enfermeiro saiba conhecer e identificar mulheres que possam estar mais vulneráveis a sofrer tais ações. Assim, o enfermeiro obstetra atuante na atenção primária à saúde e prénatal tem o dever de analisar as possibilidades. A enfermagem obstétrica é considerada a mais preparada para essas mudanças em relação a VO de acordo com a Organização Mundial de Saúde, e para uma consolidação na assistência segura no parto e no nascimento.

Logo, o objetivo da pesquisa foi buscar na literatura o que as evidências científicas abordam sobre os tipos de violências obstétricas e o papel do enfermeiro.

\section{MÉTODOS}

Trata-se de um estudo do tipo revisão integrativa da literatura desenvolvido a partir da seguinte questão norteadora: O que as evidências científicas apontam sobre a violência obstétrica e o papel do enfermeiro para minimizar os casos? A metodologia utilizada para a pesquisa foi uma revisão integrativa da literatura, que tem como alvo sintetizar informações baseando-se nas técnicas de coleta de dados de pesquisas bibliográficas, permitindo uma análise sobre a assistência de enfermagem à mulher vítima de violência obstétrica, contribuindo para a prática de enfermagem baseada em evidências científicas (MENDES KDS, et al., 2008).

Para a coleta de dados foram utilizadas as bases: Literatura Latino-americana e do Caribe em Ciências da Saúde (LILACS), Sistema Online de Busca e Análise de Literatura Médica (MEDLINE) e Acervo+. Os descritores selecionados para guiar esta busca foram: "assistência de enfermagem", "prática de enfermagem" combinados às palavras: "violência obstétrica", utilizando o operador booleano "AND". Foi usado como estratégia de busca as duas combinações mencionadas junto com a palavra "violência obstétrica" como título, resumo e assunto. Inicialmente foram encontrados 19 artigos e foram excluídos 2 estudos por duplicata. Após a leitura dos títulos e resumos foram excluídas 2 pesquisas. Após a leitura na íntegra dos estudos, 4 foram excluídos. O corpus da análise foi de 11 artigos.

Foi considerado como critério de inclusão pesquisas publicadas nos últimos 5 anos (2016-2021), que tratassem diretamente do tema violência obstétrica, com textos disponíveis na íntegra e gratuitamente, nos idiomas português e inglês. Foram selecionados artigos que procuravam explicar sobre as violências obstétricas, os tipos de violências e o enfermeiro em cada termo, além de uma busca em como o enfermeiro poderia minimizar os acontecimentos.

A leitura seletiva dos artigos foi realizada, inicialmente, com análise do título e resumo. Os artigos duplicados foram registrados apenas uma vez. Para garantir o registro conjunto de informações relevantes ao tema foi utilizado instrumento próprio para análise dos artigos, o qual incluía dados de identificação (período publicado, formação e instituição à qual os autores estão vinculados, idioma e país), aspectos metodológicos dos estudos (delineamento da pesquisa, amostragem e tratamento dos dados) e principais resultados e conclusões.

Em seguida, realizou-se análise detalhada dos artigos, considerando o rigor e as características dos mesmos, seguindo-se o registro das informações elencadas no instrumento. As informações foram interpretadas, resumidas e organizadas em quadro síntese, comparando-se os resultados, e finalizadas com proposições acerca dos estudos. A partir da análise foi possível extrair os conceitos delineados em cada artigo para a construção do estudo proposto. Desse modo, os trabalhos foram agrupados e comparados pela relevância de similaridade dos conteúdos.

\section{RESULTADOS E DISCUSSÃO}

A leitura seletiva dos 19 artigos foi realizada, inicialmente, com análise do título e resumo foram excluídos 2 estudos por duplicata (Quadro 1). Em seguida, foram excluídos 2 estudos após a leitura de títulos e resumos. Desta forma, 15 estudos foram lidos na íntegra. Foram excluídos 4 artigos por não atenderem à questão norteadora. $O$ corpus da análise foi de 11 estudos selecionados (Figura 1). 
Figura 1 - Descrição da busca realizada nas bases de dados escolhidas para a composição dos resultados da revisão integrativa, 2021.
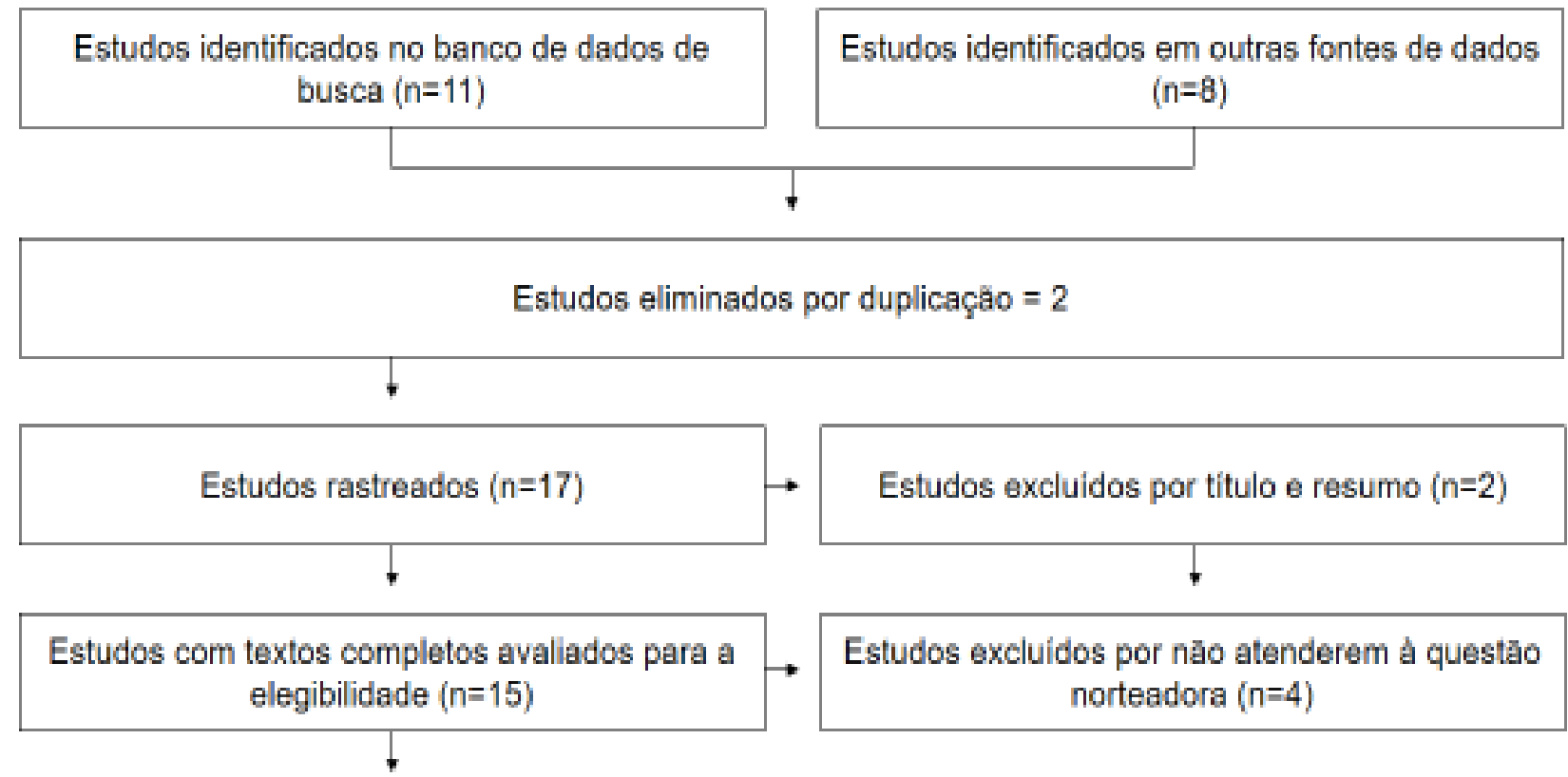

Estudos incluidos na revisăo $(n=11)$

Fonte: Souza JPS, et al., 2021.

Quadro 1 - Síntese dos artigos selecionados na revisão integrativa, 2021.

\begin{tabular}{|c|c|c|}
\hline Autores (Ano) & Título do Trabalho & Periódico \\
\hline Souza ACAT, et al. (2020) & Violência obstétrica: uma revisão integrativa. & $\begin{array}{l}\text { Revista } \\
\text { UERJ }\end{array}$ \\
\hline Miranda FL, et al. (2019) & $\begin{array}{l}\text { Violência obstétrica: percepções de enfermeiros } \\
\text { obstétricos em uma maternidade de Minas } \\
\text { Gerais }\end{array}$ & Revista HU \\
\hline Silva TM, et al. (2020) & $\begin{array}{l}\text { Violência obstétrica: a abordagem da temática na } \\
\text { formação de enfermeiros obstétricos }\end{array}$ & Acta Paul. Enferm. \\
\hline Moura RCM, et al. (2019) & $\begin{array}{l}\text { Cuidados de enfermagem na prevenção da } \\
\text { violência obstétrica. }\end{array}$ & Enfermagem em Foco \\
\hline $\begin{array}{l}\text { Martins AC, Barros GM. } \\
\text { (2016) }\end{array}$ & $\begin{array}{l}\text { Will you give birth in pain? Integrative review of } \\
\text { obstetric violence in Brazilian public units. }\end{array}$ & Revista Dor \\
\hline Zanardo GLP, et al. (2017) & $\begin{array}{l}\text { Violência obstétrica no brasil: uma revisão } \\
\text { narrativa. }\end{array}$ & Psicologia \& Sociedade \\
\hline Menezes FR, et al. (2020) & $\begin{array}{l}\text { O olhar de residentes em Enfermagem } \\
\text { Obstétrica para o contexto da violência obstétrica } \\
\text { nas instituições. }\end{array}$ & $\begin{array}{l}\text { Interface } \\
\text { Comunicação, Saúde, } \\
\text { Educação }\end{array}$ \\
\hline Lemos TAB, et al. (2019) & $\begin{array}{l}\text { Humanização como forma de superação da } \\
\text { violência obstétrica: papel do enfermeiro. }\end{array}$ & $\begin{array}{l}\text { Revista Eletrônica } \\
\text { Acervo Saúde }\end{array}$ \\
\hline Silva WB, et al. (2019) & $\begin{array}{l}\text { Educação em saúde acerca da prevenção da } \\
\text { violência obstétrica: relato de experiência. }\end{array}$ & $\begin{array}{l}\text { Revista Eletrônica } \\
\text { Acervo Saúde }\end{array}$ \\
\hline $\begin{array}{l}\text { Cavalheiro EAM, et al. } \\
(2021)\end{array}$ & Violência obstétrica: revisão de literatura. & Revista Artigos.Com \\
\hline Costa NY, et al. (2020) & $\begin{array}{l}\text { O pré-natal como estratégia de prevenção a } \\
\text { violência obstétrica. }\end{array}$ & $\begin{array}{l}\text { Revista Eletrônica } \\
\text { Acervo Saúde }\end{array}$ \\
\hline
\end{tabular}

Fonte: Souza JPS, et al., 2021. 
De acordo com os estudos a VO está presente no pré-parto, parto e pós-parto, pelos profissionais de saúde que a ajudam, logo a VO é definida como qualquer atitude, desrespeitosa e desumana, além de negligência e maus tratos a parturiente e seu recém-nascido (MOURA RCM, et al., 2019).

Ao longo do estudo Souza ACAT, et al. (2020), relata que a VO pode ser praticada envolvendo ações por parte dos serviços de saúde como mau atendimento, falta de explicação das intervenções e procedimentos realizados, falta de comunicação e elo profissional/paciente, uso de frases ofensivas, repreensões, ameaças contra as mulheres e seus bebês no momento do parto.

Categorizando os tipos de VO sofridos pelas parturientes identificam-se violência institucional como ausência de estrutura adequada, recursos físicos e humanos precários; A violência moral sendo associada as condutas profissionais que desvalorizam a autonomia e o saber da parturiente sobre o seu corpo; $\mathrm{Na}$ violência física e mostrado a utilização inadequada e desnecessária da tecnológica como a episiotomia, manobra de Kristeller e o uso da ocitocina; Na violência sexual é apresentado as falas de baixo escalão e piadas de conteúdo sexual sobre a mulher naquele momento, podendo ser incluído também na violência psicológica e verbal que vai muito além como os xingamentos, julgamentos, gritos, humilhação através das palavras, expressões de ironia, comentários desrespeitosos e ameaças de abandono (MARTINS AC e BARROS GM, 2016).

É abordado os comentários com intuito de insultar e depreciar, além de piadas pelos profissionais, como exemplos citando que "Na hora de fazer não chorou, está chorando agora por quê?"ou "ah, não chora não, ano que vem você está aqui de novo", e quando o caso é um paciente em situação de aborto que na hora de examinar escuta: "Quem quer um feto morto?" mostra-se casos de violência psicológica e verbal (SOUZA ACAT, et al., 2020).

Em várias instituições é mostrado os atendimentos abusivos como as intervenções cirúrgicas, a falta de informação e até a negação ao direito ao acompanhante que é um direito garantido em lei na Lei do Acompanhante: Lei no 11.108/2005, a presença do acompanhante ainda é permitida e quando a aceitam fazem restrição quanto a escolha do gênero, sendo uma violação da lei que assegura a presença do acompanhante e de a mulher ter o direito em escolher seu acompanhante durante o parto e pós-parto (ZANARDO GLP, et al., 2017).

A tricotomia, privação de alimento, administração deliberada de ocitocina, amniotomia de rotina, adoção da posição supina, sendo que a mulher tem que ter a liberdade de escolher como que parir, manobra de Kristeller para acelerar o nascimento do bebê mas que traz risco para os dois, e a episiotomia de rotina também são identificadas como práticas prejudiciais e que devem ser eliminadas ou que não devem ser estimuladas, por não existir evidências suficientes (SOUZA ACAT, et al., 2020).

O estudo de Miranda FL, et al. (2019), apresenta resultados que destacam nas assistências ao parto condutas inadequadas, procedimentos desnecessários com fins didáticas e complicações causadas por tratamento médico, além de preconceito de gênero, raça/etnia e de classe socioeconômica. Em relação às condutas, destacam-se a utilização de discursos ríspidos, tratamento impaciente com a parturiente, ameaças de abandonar à mulher durante o trabalho de parto por não ter a cooperação da mesma e comentários ofensivos e depreciativos, caracterizando a violência verbal e psicológica.

Foi citado na pesquisa por Menezes FR, et al. (2020), o déficit nos meios para identificar e notificar VO fazendo com que seja ignorando todas as práticas usadas. Os problemas para atender nos serviços de denúncia piora no processo trazendo assim a importância da tecnologia onde a estratégia de mobilização social é utilizada pelos meios de informação também usado como ferramenta de empoderamento feminino e participação política e fazendo fortalecer a voz dos usuários como por exemplo as mulheres vítimas de VO.

De acordo com o estudo Silva TM, et al. (2020), aponta a importância do reconhecimento judicial por conta que as mulheres lesadas nem sempre sabem como procurar reparação, de modo que através do enfermeiro pode assim acontecer um o diálogo permitindo medidas de prevenção e informação por isso é tão importante um atendimento humanizado com o enfermeiro obstétrico qualificado para melhorar a qualidade do serviço e contribuir de forma mais humana e positiva. 
No estudo Silva TM, et al. (2020), mostra que de acordo com o discurso dos profissionais, foi identificado possíveis riscos ao feto por conta da falta de cuidado. Contudo, mostra que os problemas que ocorrem por parte da equipe de profissionais por conta do acúmulo de trabalho como alta rotatividade e forma precária de trabalhos, e responsabilidades além da que foi contratada.

De acordo com o estudo esses tipos de violência ainda existem, muitas vezes por conta das dificuldades enfrentadas no dia a dia das instituições, como exemplo a falta de estrutura em relação a demanda que aceita, a sobrecarga dos profissionais e a falta de preparo para o mesmo, além dos problemas como falta de materiais, além da falha na assistência e organização da instituição (MENEZES FR, et al., 2020).

A mudança que a enfermagem faz nesse tema é bem abrangente e exerce um papel fundamental no processo de humanização, minimizando técnicas invasivas que podem levar a inúmeras complicações. Esse processo de humanização busca resgatar a humanidade e individualidade da mulher fazendo o oposto de que a mulher é vista apenas como uma fábrica de bebês. $O$ conceito de humanização segue pregando atitudes com o objetivo de promover partos e nascimentos saudáveis e colocar a mulher como protagonista, ofertando procedimentos que tragam benefícios além da sua privacidade e autonomia (LEMOS TAB, et al., 2019).

Para isso mostra-se necessário o fortalecimento da enfermagem obstétrica a fim de evitar a reprodução do modelo tecnicista que ainda resiste. Uma maior atuação de enfermeiras gerou reduções em algumas práticas como do uso de anestesia peridural, de episiotomia e de parto instrumental (com fórceps ou vácuo extrator), bem como, maior probabilidade de parto vaginal espontâneo e do aleitamento materno imediato, logo após o nascimento e, redução do número de cesáreas sem indicação real contribuindo para o sucesso no parto e pós-parto além da saúde da mãe e o seu bebê. Nos Centros de Parto Normais (CPN) a parturiente tem total liberdade em como quer seu parto, além do seu acompanhante e uma doula, favorecendo práticas saudáveis e naturais além da liberdade da mulher, minimizando intervenções obstétricas desnecessárias (SOUZA ACAT, et al., 2020).

A falta de informação e de esclarecimentos sobre os processos intervenções e autorização sobre a parturiente, permite que os profissionais realizem procedimentos que infrinjam e desrespeitam os direitos sexuais e reprodutivos da mulher além da sua própria autonomia no trabalho de parto. Destaca-se então a importância dos profissionais de enfermagem em praticar a educação em saúde de forma que as informações a serem repassadas à mulher possam de fato trazer empoderamento a ela, especialmente no que tange ao seu corpo e minimização de práticas assistenciais inadequadas (SILVA WB, et al., 2019).

Foi abordado a quantidade de exemplos de VO por conta que nem todas as gestantes são orientadas sobre as violências obstétricas pelos profissionais durante o pré-natal e o que fazer em caso de seu médico praticá-la e o enfermeiro desde o início pode participar para evitar acontecimentos como esses (CAVALHEIRO EAM, et al., 2021). Com todos os estudos e exemplos abordados de VO como práticas educativas a educação em saúde realizada durante o acompanhamento pré-natal possibilita o esclarecimento de dúvidas, troca de vivências, criação de vínculo e diálogo entre o profissional enfermeiro, a gestante e sua família, gerando aprendizado crítico para enfrentamento das adversidades, além de sedimentar os direitos e deveres como cidadãos práticas vividas na UBS com enfermeiras obstétricas (COSTA NY, et al., 2020).

Contudo, o combate a prática da VO dar-se através da garantia dos direitos as gestantes como por exemplo uma assistência de qualidade ao pré-natal, pré-parto, parto, puerpério e em situações de aborto, garantindo a humanização em todas as etapas. Salientando também que essa assistência deve-se incluir benefícios a gestante como a sua autonomia além de compromisso, respeito, empatia da parte da equipe e os profissionais (LEMOS TAB, et al., 2019).

\section{CONSIDERAÇÕES FINAIS}

Os estudos relatam os tipos de VO sofridas pela mulher durante o trabalho de parto como mau atendimento nos serviços de saúde, a falta de explicação das intervenções e procedimentos realizados, falta de comunicação e elo profissional/paciente; o uso de frases ofensivas, repreensões, ameaças contra as 
mulheres e seus bebês no momento do parto, utilização de jargões pejorativos e piadas. Destaca-se a importância de buscar mudanças na tratativa da obstetrícia, pois de acordo com o estudo há indícios de práticas à parturiente de caráter duvidosas, no entanto, é a área da saúde que mostrou um interesse em melhorar a assistência buscando avanço nos estudos e debatendo que a informação é essencial para esse processo.

\section{REFERÊNCIAS}

1. BRANDT GP, et al. Violência obstétrica: a verdadeira dor do parto. RGS, 2018; 19(1): 19-37.

2. BRASIL. Manual do Ministérios de Saúde. 2016. Disponível em: http://biblioteca.cofen.gov.br/partonormal/\#: :text=Sintetiza\%20e\%20avalia\%20sistematicamente\%20a,e\%20incentivar\%200\%20parto\%20normal.. Acesso em: 1 de junho de 2021.

3. CAVALHEIRO EAM, et al. Violência obstétrica: revisão de literatura. Revista Artigos. Com, 2021; 26 : e6695.

4. COSTA NY, et al. O pré-natal como estratégia de prevenção a violência obstétrica. Revista Eletrônica Acervo Saúde, 2020; 12(12): e4929.

5. LEMOS TAB, et al. Humanização como forma de superação da violência obstétrica: papel do enfermeiro. Revista Eletrônica Acervo Saúde, 2019; 23: e207.

6. MARTINS AC, BARROS GM. Will you give birth in pain? Integrative review of obstetric violence in Brazilian public units. Revista Dor, 2016; 17: 3.

7. MENDES KDS, et al. Integrative literature review: a research method to ncorporate evidence in health care and nursing. Texto Contexto Enferm, 2008; 17(4): 758-64.

8. MENEZES FR, et al. O olhar de residentes em Enfermagem Obstétrica para o contexto da violência obstétrica nas instituições. Interface - Comunicação, Saúde, Educação, 2020.

9. MIRANDA FL, et al. Violência obstétrica: percepções de enfermeiros obstétricos em uma maternidade de Minas Gerais; HU Rev, 2019; 45(4): 415-420.

10. MOURA RCM, et al. Cuidados de enfermagem na prevenção da violência obstétrica. Enferma em Foco, $2019 ; 9: 4$.

11. RIBEIRO KG, et al. Caracterização da violência obstétrica na produção científica: uma revisão integrativa. Revista Eletrônica Acervo Saúde, 2021; 13(4): e6604.

12. SILVA TM, et al. Violência obstétrica: a abordagem da temática na formação de enfermeiros obstétricos. Acta Paulista de Enfermagem, 2020; 33.

13. SILVA WB, et al. Educação em saúde acerca da prevenção da violência obstétrica: relato de experiência. Revista Eletrônica Acervo Saúde, 2019; 14: e1163.

14. SOUZA A, et al. Fatores associados à ocorrência de violência obstétrica institucional: uma revisão integrativa da literatura. Revista de Ciências Médicas, 2017; 25: 115.

15. SOUZA ACAT, et al. Violência obstétrica: uma revisão integrativa. Revista Enfermagem UERJ, 2020 ; e45746.

16. ZANARDO GLP, et al. Violência obstétrica no Brasil: uma revisão narrativa. Psicologia \& Sociedade, $2017 ; 29$. 